Electronic Supplementary Information for:

\title{
Influence of TMPO Loading on the Measurement of Zeolite Acidity by Solid-State NMR Spectroscopy
}

Yongxiang Wang, ${ }^{\mathrm{a}, \mathrm{b}} \uparrow$ Shaohui Xin, ${ }^{\mathrm{a}, \mathrm{c}, \mathrm{d} \dagger}$ Yueying Chu, ${ }^{\mathrm{a} *} \mathrm{Jun} \mathrm{Xu},{ }^{\mathrm{a}}$ Guodong Qi, ${ }^{\mathrm{a}}$ Qiang Wang, ${ }^{a *}$ Qinghua Xia ${ }^{b}$ and Feng Denga*

aNational Centre for Magnetic Resonance in Wuhan, State Key Laboratory of Magnetic Resonance and Atomic and Molecular Physics, Wuhan Institute of Physics and Mathematics, Innovation Academy for Precision Measurement Science and Technology, Chinese Academy of Sciences, Wuhan 430071, China.

${ }^{b}$ Ministry-of-Education Key Laboratory for the Synthesis and Application of Organic Functional Molecules, Hubei University, Wuhan 430062, P. R. China.

'University of Chinese Academy of Sciences, Beijing 100049, P. R. China.

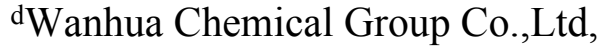

Email: chuyueying@wipm.ac.cn; qiangwang@wipm.ac.cn; dengf@wipm.ac.cn

\section{Author Contributions}

$\dagger$ These authors equally contribute to this work 


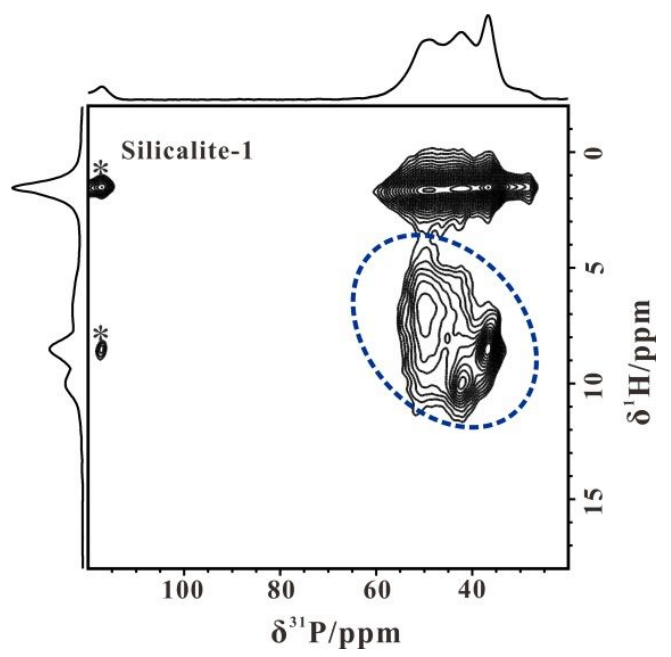

Figure S1. 2D ${ }^{31} \mathrm{P}\left\{{ }^{1} \mathrm{H}\right\}$ CP HETCOR NMR spectrum of silicalite-1 zeolite with saturated TMPO loading. 